\title{
Mapping of the complement C9 binding domain on Trichinella spiralis paramyosin
}

\author{
Xi Zhao, Yuwan Hao, Jing Yang, Yuan Gu and Xinping Zhu*
}

\begin{abstract}
Background: Trichinellosis is an important foodborne zoonosis that is distributed worldwide. Trichinella spiralis may evade host complement-mediated attack by expressing complement inhibitory proteins, such as paramyosin (Pmy). Previous studies have shown that Trichinella spiralis paramyosin (Ts-Pmy) is able to bind to the human complement component C9 to inhibit the complement activation and protect the parasite from complement-mediated attack. Further determination of the complement-binding domain on Ts-pmy will enable us to better understand the Ts-Pmy's biofunction in the immune evasion and provide feasible approach to develop epitope-based subunit vaccine against trichinellosis.

Methods: The complement C9 binding region on Ts-Pmy was determined by expression of overlapped fragments of Ts-Pmy and their binding activities to C9. The exact binding site was further narrowed-down to a 14-amino acid peptide at C-terminus using synthesized peptides with different size of amino acid sequence. The C9 complement-binding of the 14-amino acid peptide and its interference in the C9 polymerization and the complement-mediated lysis of rabbit erythrocytes was investigated.

Results: The protein interaction between human C9 and native Ts-Pmy was further confirmed by immunoprecipitation with $T$. spiralis lysates. The fragmental expression and C9 binding assays identified that the binding region of Ts-Pmy to C9 is located within 831-885 of Ts-Pmy C-terminus. The exact binding site on Ts-Pmy to C9 was narrowed down to 14 amino acid residues $\left({ }^{866} \mathrm{Val}-{ }^{879} \mathrm{Met}\right)$ by using different sizes of synthesized peptides. In the presence of the synthesized 14-amino acid peptide, human C9 polymerization and the hemolytic activity of the human complement was inhibited.
\end{abstract}

Conclusions: Our results revealed the precise molecular basis for T. spiralis to produce Ts-Pmy as an immunomodulator to evade the attack of the host complement system as a survival mechanism.

Keywords: Trichinella spiralis, Paramyosin, Immune evasion, Complement C9, Binding domain

\section{Background}

Trichinellosis is a globally widespread foodborne zoonosis that occurs by ingesting raw or undercooked meat of infected animals that contain parasitic larvae [1]. Muscle larvae (ML) are released from muscle tissue by digestive enzymes in the stomach and migrate to the small intestine, where the larvae develop into adult worms (AD). Adult females produce newborn larvae (NBL), which then penetrate into the mesenteric lymphatic vessels or the bloodstream and spread throughout the body. Migrating NBL leave the capillaries and finally

\footnotetext{
* Correspondence: zhuxping@ccmu.edu.cn

Department of Parasitology, School of Basic Medical Sciences, Capital Medical University, Beijing, China
}

invade into muscle tissue, where the NBL develop to $\mathrm{ML}$ and encapsulate in individual skeletal muscle cells [2].

Trichinella spiralis is the most common species that infects human and mammalian hosts, such as pigs [3]. Human trichinellosis is characterized by high fever, facial edema and myositis, which could be serious, particularly in elderly patients [4]. Being regarded as an emerging or re-emerging disease in some parts of the world due to changes in diet and cooking practices [3,5], trichinellosis is not only a public health hazard but also an economic problem for livestock production and food safety [6,7]. Consequently, there is an urgent requirement for developing therapeutic and preventive vaccines to control the infection [8]. 
The complement system represents a cornerstone of the innate defense against infection and provides a vital first line of defense against invading pathogens [9]. Among the complement-evasion strategies to escape the host immune attack, the capture of host complement components on the parasite surface and then inactivating their functions is an evasion mechanism that is frequently adopted by many parasites during the establishment of parasitism [10]. Similar to other parasitic helminthes, $T$. spiralis utilizes molecules or structures on the outermost cuticle/epicuticle to bind complement components, such as C1q, C3, C5, C8 and C9 [11-13], to evade the complement attachment by inhibiting the formation of the membrane attack complex (MAC) [13].

Paramyosin is a dimeric fibrillar protein that forms the thick myofilaments of invertebrate muscle. In our previous study, a full-length cDNA encoding $T$. spiralis paramyosin (Ts-Pmy) was cloned by immunoscreening an adult worm cDNA library using $T$. spiralis-infected rabbit sera [14]. $\mathrm{BALB} / \mathrm{c}$ mice vaccinated with recombinant $T s$-Pmy (rTsPmy) developed a Th1/2 mixed immune response and were partially protected against a $T$. spiralis larval challenge $[14,15]$. Subsequently, the expression of Ts-Pmy was observed on the outer membrane of newborn larvae and adult worms using immunogold electron microscopy and immunofluorescence staining [16]. A functional analysis identified that rTs-Pmy was able to bind to the human complement components $\mathrm{C} 8$ and $\mathrm{C}$, which consequently inhibits the formation of MAC and thereby protects the parasite from being attacked by activated complement [16]. However, the complement binding site on Ts-Pmy has not been determined. In the present study, we expressed different fragments of $T s$-Pmy, characterized the interaction between different fragments of Ts-Pmy and the complement component C9 and finally pinpointed the complement binding site on $T s$-Pmy within the region from ${ }^{866} \mathrm{Val}$ to ${ }^{879}$ Met at the $\mathrm{C}$-terminus.

\section{Methods}

\section{Ethics statement}

Experimental animals were purchased from the Laboratory Animal Services Center of Capital Medical University (Beijing, China). Experimental procedures were reviewed and approved by the Capital Medical University Animal Care and Use Committee and were consistent with the NIH Guide for the Care and Use of Laboratory Animals.

\section{Parasites and antigen preparation}

T. spiralis of ISS 533 strain was maintained in female ICR mice and $T$. spiralis ML were recovered from the muscles of infected mice by a standard pepsin/hydrochloric acid digestion method [17]. Adult worms were collected from the intestines of an infected Wistar rat. Crude somatic extracts of adult worms were prepared by homogenizing the worms in $1 \times$ PBS, $\mathrm{pH} 7.4$ and centrifuging at $16,000 \times \mathrm{g}$. The protein concentration of the extracts was determined by BCA assay kit (Merck, Germany).

\section{Immunoprecipitation}

To further verify the protein interaction between the human complement C9 and the native Ts-Pmy, immunoprecipitation was performed with $T$. spiralis adult extracts as described previously [18]. Protein G MicroBeads (Miltenyi Biotec, Germany) were pre-incubated with $3 \mu \mathrm{g}$ of human C9 (Merck, Germany) and $2 \mu \mathrm{g}$ of anti-C9 mAb (IgG1, Abnova, Taiwan) for $30 \mathrm{~min}$ on ice. In total, $40 \mu \mathrm{g}$ of T. spiralis adult extracts was then added and incubated overnight at $4^{\circ} \mathrm{C}$ with rotation. Beads were washed four times with washing buffers (1\% NP40, $50 \mathrm{mM}$ Tris buffer, $\mathrm{pH}$ 8.0) before being added with pre-heated $1 \times$ SDS gel loading buffer to elute proteins. Samples were separated by SDS-PAGE and probed with anti-Ts-Pmy mAb (7E2) [19]. IRDye $800 \mathrm{CW}$-labeled goat anti-mouse IgG (LI-COR, Germany) was used as the secondary antibody.

\section{Fragmental expressions of Ts-Pmy in E. coli}

To identify and locate the binding site of Ts-Pmy that binds to the human complement $\mathrm{C}$, the DNA encoding the $\mathrm{N}$ terminus (Ts-Pmy1-315), C-terminus (Ts-Pmy571-885) and middle region (Ts-Pmy286-600) of $T$. spiralis paramyosin with 30 amino acids overlapped were cloned into the bacterial expression vector pET28a, with 6-histidine expressed at the N-terminus as tags (Merck, Germany). To further locate the binding site at the C-terminus of $T s$-Pmy, the DNA encoding the fragments of Ts-Pmy571-695, TsPmy666-790, Ts-Pmy761-885, Ts-Pmy761-815, Ts-Pmy796850 and $T s$-Pmy831-885 were subcloned into pET28a to express the fragments of $T s$-Pmy C-terminus. The recombinant plasmids with sequencing confirmed right reading frames were transformed into competent E. coli BL21 pLysS cells, and the recombinant $T$ s-Pmy fragments were induced with $1 \mathrm{mM}$ IPTG at $37^{\circ} \mathrm{C}$ for $6 \mathrm{~h}$. The expressed fragments were purified from the induced bacterial lysates using Ni-charged His-Bind columns (Merck, Germany). The purified fragments were examined by Western blot with a monoclonal anti-HisTag antibody $(0.2 \mu \mathrm{g} / \mathrm{ml}$; Merck, Germany).

\section{Peptide synthesis}

To finally determine the amino acid sequence that binds to $\mathrm{C}$, the peptides with different amino acid sequences within the $\mathrm{C} 9$ binding region $\left({ }^{831} \mathrm{Leu}\right.$ to ${ }^{885} \mathrm{Tyr}$ ) were synthesized by solid-phase peptide synthesis (Aviva Bio, China). The obtained peptides were purified up to $95 \%$ by preparative RP-HPLC and verified by mass spectrometry. 


\section{Binding assay of rTs-Pmy fragments and synthesized peptides to human $\mathrm{C} 9$}

To determine whether the expressed recombinant $T s$ Pmy fragments bind to human C9, the fragments and non-relevant control BSA (Sigma, USA) (2 $\mu \mathrm{g}$ each) were subjected to SDS-PAGE under reducing conditions and then transferred to a nitrocellulose membrane. After blocking with $5 \%$ milk in PBS, the membrane was incubated with human C9 (Merck, Germany) $(1 \mu \mathrm{g} / \mathrm{ml})$ at $37^{\circ} \mathrm{C}$ for $2 \mathrm{~h}$ and then probed with anti-C9 mAb $(0.2 \mu \mathrm{g} / \mathrm{ml}$; Abnova, Taiwan) at room temperature for $1 \mathrm{~h}$. IRDye $800 \mathrm{CW}$-labeled goat anti-mouse IgG (50 ng/ml; LI-COR, Germany) was used as the secondary antibody.

To determine whether synthesized peptides bind to human $\mathrm{C}$, the peptides and non-relevant control BSA (Sigma-Aldrich, USA) (5 $\mu \mathrm{g}$ in $2.5 \mu \mathrm{l}$ each) were spotted onto a nitrocellulose membrane using a narrow-mouth pipette tip. After blocking with $1 \%$ BSA in PBS, the membrane was incubated with human $C 9(1 \mu \mathrm{g} / \mathrm{ml})$ at $37^{\circ} \mathrm{C}$ for $2 \mathrm{~h}$ and then with anti-C9 $\mathrm{mAb}(0.2 \mu \mathrm{g} / \mathrm{ml})$ at room temperature for $1 \mathrm{~h}$.

All membranes mentioned above were detected and imaged using an Odyssey infrared imaging system (LI-COR, Germany).

\section{C9 polymerization assays}

To determine the effect of the synthesized peptide on C9 polymerization, human C9 $(3 \mu \mathrm{g})$ was pre-incubated with the synthesized peptide at $37^{\circ} \mathrm{C}$ for $40 \mathrm{~min}$ and

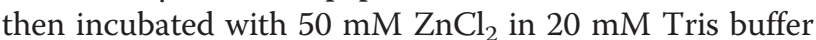
$(\mathrm{pH} 7.2)$ at $37^{\circ} \mathrm{C}$ for $2 \mathrm{~h}$ [20]. $\mathrm{Zn}^{2+}$-induced polymerized C9 (polyC9) is resistant to dissociation by boiling in $1 \%$ SDS and, thus, could be detected by SDS-PAGE [21]. The inhibition of C9 polymerization was shown by SDSPAGE on a 3 to $20 \%$ acrylamide gradient gel under reducing conditions and stained with Coomassie blue. The full-length rTs-Pmy was added as a positive control. Recombinant Ts87 (rTs87), a specific T. spiralis secreted protein [22], was used as a non-relevant control.

\section{Hemolytic assay of the alternative complement pathway}

The complement-mediated lysis of rabbit erythrocytes $\left(E_{R}\right)$ was performed via the alternative pathway of complement activation [12]. To identify whether the synthesized peptide acts as an inhibitor or a neutralizer of the complement-mediated lysis activated by the alternative pathway, $100 \mu \mathrm{l}$ of fresh normal human serum (NHS) was pre-incubated with various amounts of synthesized peptide or rTs-Pmy in Mg-EGTA solution (5 $\mathrm{mM} \mathrm{MgCl}_{2}$, $10 \mathrm{mM}$ EGTA) for $30 \mathrm{~min}$ before being added into freshly prepared $E_{R}\left(1 \times 10^{8}\right)$ in $100 \mu$ l HBSS (Hank's Balanced Salt Solution, without calcium and magnesium, pH 7.4, Gibco, USA) at $37^{\circ} \mathrm{C}$ for $30 \mathrm{~min}$. Heat-inactivated NHS was used as a control. The hemolytic assay was stopped by adding
$1 \mathrm{ml}$ of cold HBSS that contained $10 \mathrm{mM}$ EDTA. After centrifugation at $3,000 \times \mathrm{g}$ and $4^{\circ} \mathrm{C}$ for $10 \mathrm{~min}$, the amount of hemoglobin released into the supernatant was measured at $412 \mathrm{~nm}$. The percentage of lysis (relative to cells completely lysed by water) was then calculated. rTs 87 was used as a non-relevant control.

\section{Statistical analysis}

The data were expressed as the mean \pm standard error (S.E.) and were evaluated using the software Prism 6 (GraphPad Inc., USA) with a one-way ANOVA; P <0.05 was regarded as statistically significant.

\section{Results}

\section{Binding of native Ts-Pmy to human C9}

The protein interaction between human $C 9$ and native $T s$-Pmy was further confirmed by immunoprecipitation and Western blot analysis. Ts-Pmy in the adult worm extracts was specifically recognized by anti-Ts-Pmy mAb 7E2 ( 102 kDa) (Figure 1, Lane 1). The native Ts-Pmy in the adult extracts was bound to human $\mathrm{C} 9$, and the binding complex was pulled down by anti-C9 mAb (Figure 1, Lane 3). Anti-C9 mAb alone did not bind to the native Ts-Pmy in the extracts (Figure 1, Lane 2). As shown in lanes 2 and 3, the heavy chain and light chain of anti-C9 mAb were pulled down by Protein $\mathrm{G}$ and recognized by the anti-mouse IgG secondary antibody.

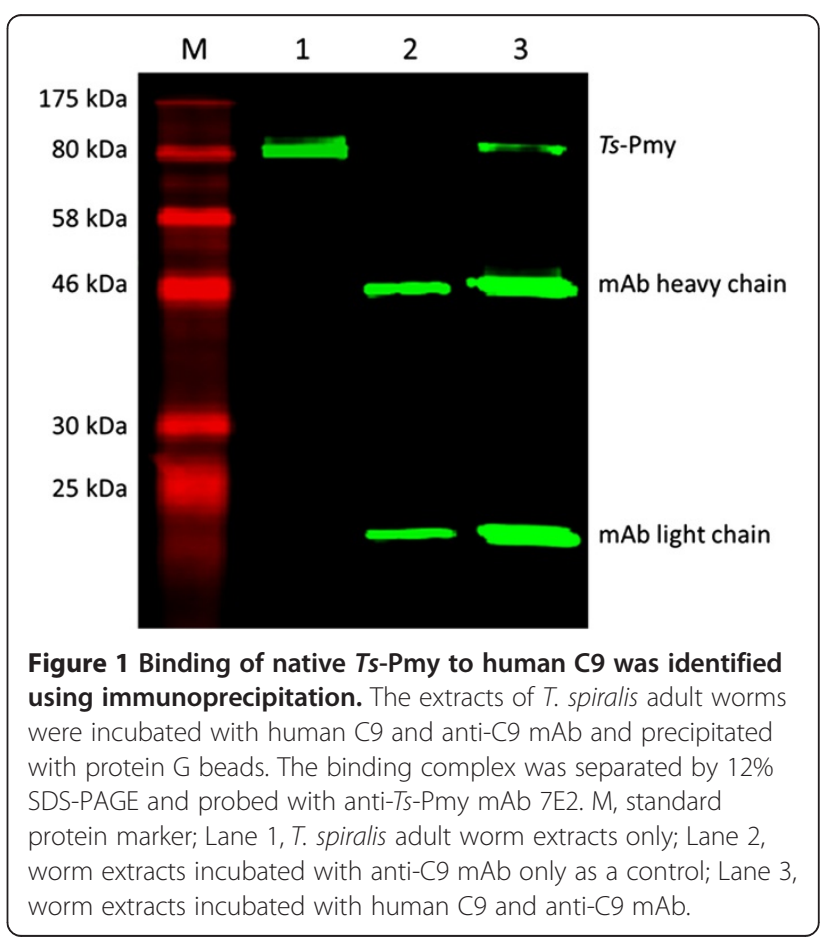




\section{Fragmental expression mapping of the Ts-Pmy binding site to human $\mathrm{C} 9$}

To identify the binding site of Ts-Pmy to human C9, different fragments covering the whole molecule with 30 amino acids overlapped were expressed as recombinant fragmental proteins (Figure 2A). The recombinant fragments were transferred to a nitrocellulose membrane, probed with human $\mathrm{C} 9$ and detected using anti-C9 mAb. The Western blot analysis demonstrated that the binding site in Ts-Pmy to human C9 was located at the C-terminus of Ts-Pmy (Ts-Pmy571-885) (Figure 2B). Further fragment expressions were performed within the $\mathrm{C}$-terminus and the binding site was narrowed down to Ts-Pmy761-885 (Figure 2C). Further pinpoint of the $\mathrm{C} 9$ binding region was determined by smaller fragment expression, which is located within Ts-Pmy831-885 (Figure 2D). As a non-relevant control, BSA did not bind to human $C 9$. All recombinant protein fragments could be recognized by anti-HisTag antibody.

\section{Determination of the C9 binding site in Ts-Pmy by synthesized peptide mapping}

To further pinpoint the $\mathrm{C} 9$ binding site within the $\mathrm{C}$ terminus of Ts-Pmy (Pmy831-885) defined by the fragment expressions, the 12 overlapped peptides covering this region were synthesized for their binding capacity to human C9 (Figure 3). After being spotted onto a nitrocellulose membrane, the synthesized peptide was probed with human $\mathrm{C} 9$ and detected with anti-C9 mAb. The Dotblot analysis demonstrated that P8 within Ts-Pmy866-880 was able to bind to human C9 (Figure 3). The exact C9 binding sequence was narrowed down to $T s$-Pmy866-879 (P10) by removing one amino acid at position 880 . The further removal of one amino acid at the $\mathrm{N}$-terminus (P11) or at the C-terminus (P12) caused the loss of the binding to human C9 (Figure 3), indicating that the sequence of the C9 binding site in Ts-Pmy was precisely narrowed down to 14 amino acid residues between ${ }^{866} \mathrm{Val}$ to ${ }^{879}$ Met that forms the binding site structure to $\mathrm{C} 9$. As a non-relevant control, BSA did not bind to human C9.

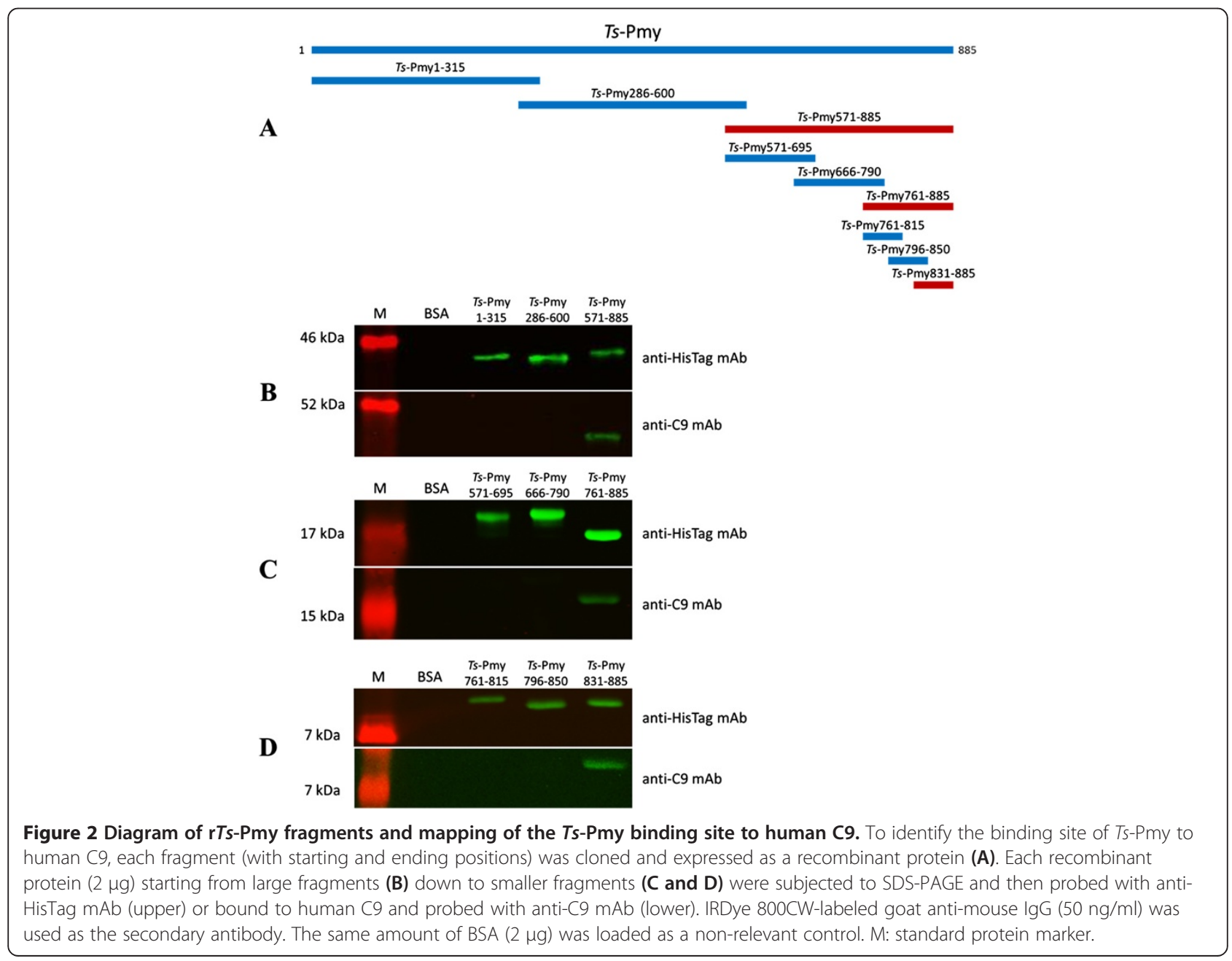




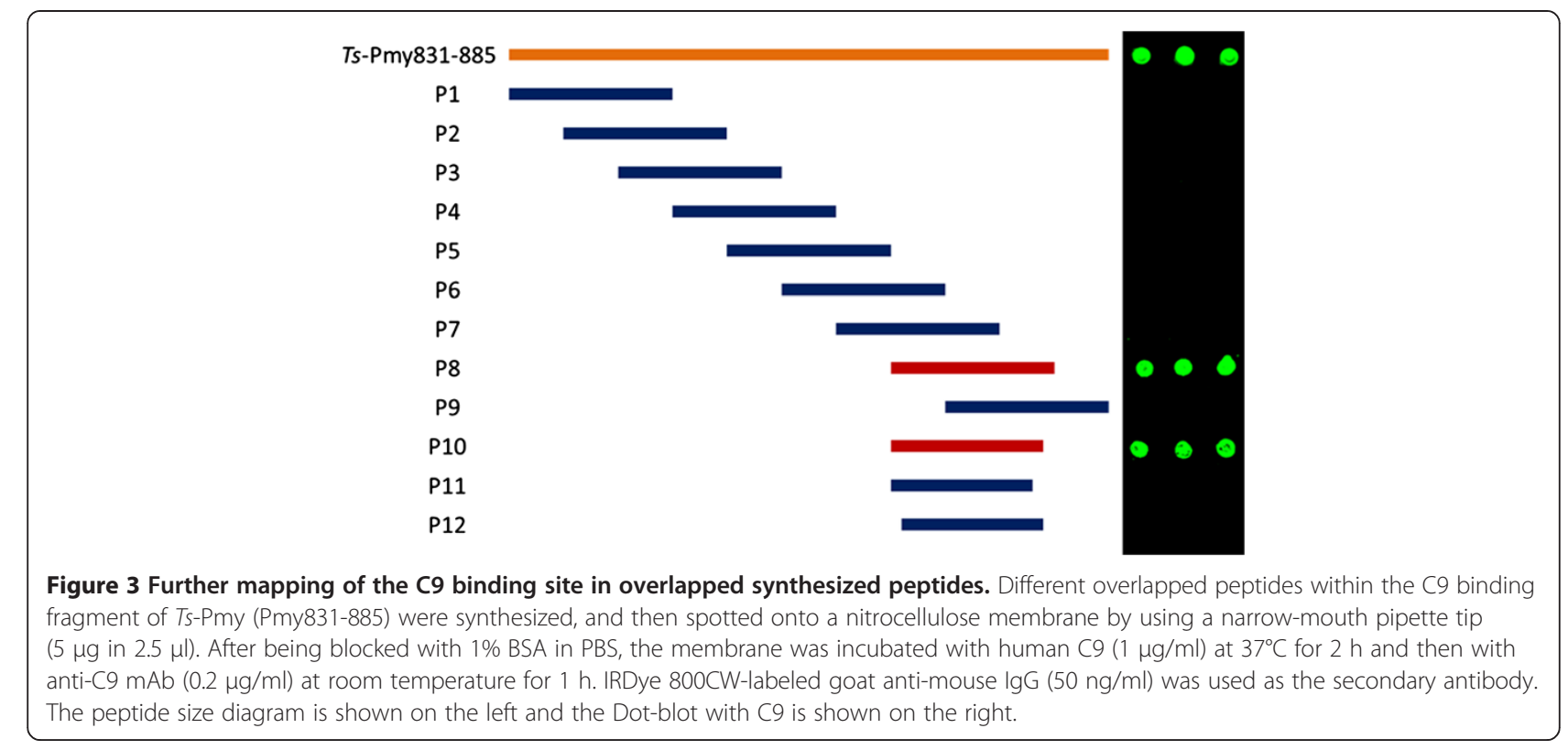

\section{Inhibition of C9 polymerization by the binding site peptide P10}

To determine whether binding peptide P10 $\left({ }^{866} \mathrm{Val}\right.$ to ${ }^{879} \mathrm{Met}$ ) inhibits the $\mathrm{Zn}^{2+}$-induced C9 polymerization as full-length $\mathrm{r} T s$-Pmy [16], C9 was mixed with different amount of peptide P10 (2.5, 5, $10 \mu \mathrm{g})$ and then incubated with $50 \mathrm{mM} \mathrm{ZnCl}$. As shown in Figure 4, peptide P10 inhibited $\mathrm{Zn}^{2+}$-induced $\mathrm{C} 9$ polymerization in a dose-dependent manner. The polymerization of $3 \mu \mathrm{g} \mathrm{hu}$ man C9 was completely inhibited by $10 \mu \mathrm{g}$ peptide P10 (Figure 4) and similarly by the full-length rTs-Pmy. rTs87 $(10 \mu \mathrm{g})$ did not inhibit C9 polymerization as a non-relevant control. Without $\mathrm{Zn}^{2+}$, the $\mathrm{C} 9$ did not form the polymer (Figure 4 ).

\section{Inhibition of complement-mediated hemolysis by the binding site peptide P10}

After incubation with different amounts of peptide P10 and rTs-Pmy $(0,5,10,20 \mu \mathrm{g})$, complement-mediated $E_{R}$ lysis with NHS via the alternative pathway was significantly inhibited in a dose-dependent manner (Figure 5), which suggested that peptide P10 bound to $\mathrm{C} 9$ and consequently inhibited the complement-mediated hemolysis. As a non-relevant control, $\mathrm{r} T s 87$ did not inhibit complementmediated hemolysis.

\section{Discussion}

The complement system has a critical role in the recognition, opsonization and elimination of pathogenic intruders. Among bacteria, viruses, fungi and parasites, many species have developed specific complement-evasion strategies to escape the attack from host's immune system. The most common complement-evasion mechanism is to capture the soluble host complement regulators or express their structural mimics on the microbial surface [10]. Similar to other pathogens, helminths have a particularly wide and diverse arsenal of complement-evasion proteins, many of which have been characterized recently [10].

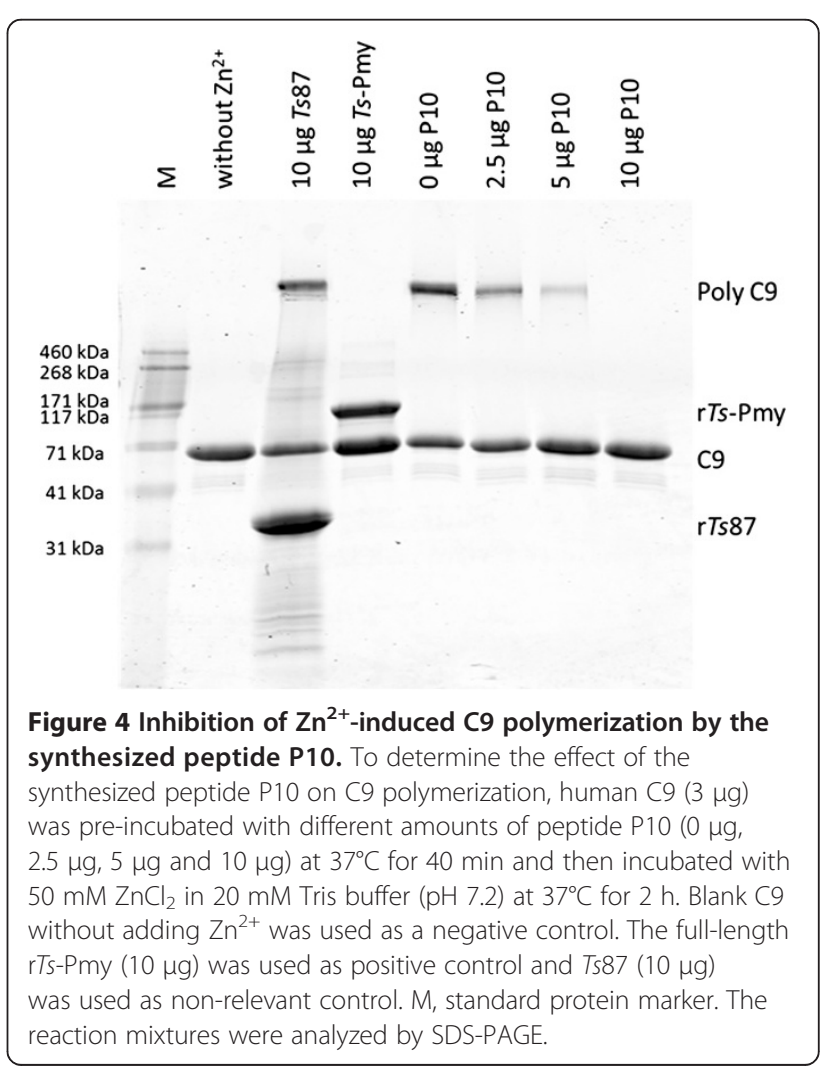




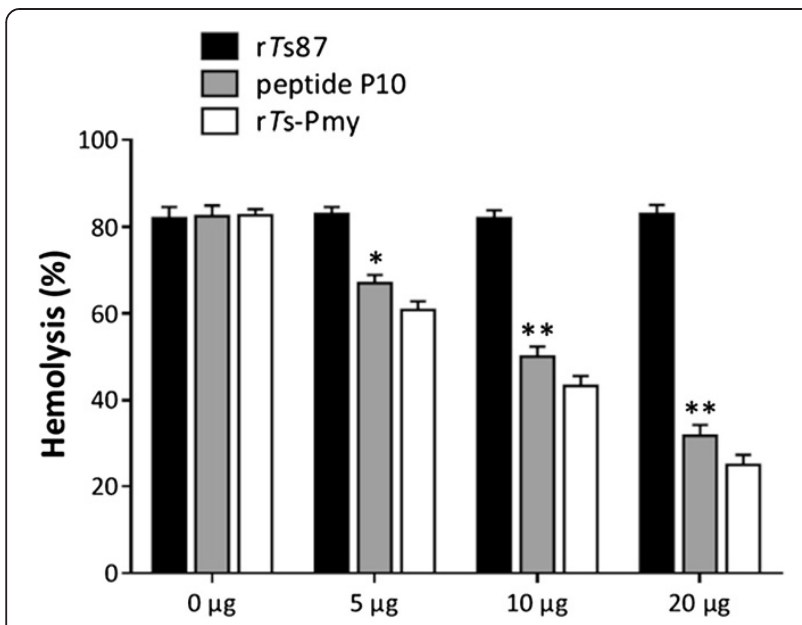

Figure 5 Inhibition of complement-mediated hemolysis by peptide P10. NHS was pre-incubated with various amounts $(0,5$, 10, $20 \mu \mathrm{g}$ ) of peptide P10 and rTs-Pmy in Mg-EGTA solution ( $5 \mathrm{mM}$ $\mathrm{MgCl}_{2}, 10 \mathrm{mM} \mathrm{EGTA}$ ) for 30 min before adding the mixture into freshly washed $E_{R}\left(1 \times 10^{8}\right)$ in $100 \mu \mathrm{l} \mathrm{HBSS}$ at $37^{\circ} \mathrm{C}$ for $30 \mathrm{~min}$. rTs 87 was used as a non-relevant control. The amount of hemoglobin released into the supernatant was measured at $412 \mathrm{~nm}$, and the percent lysis was calculated compared to the complete lysis (water) The results are shown as the means \pm SE for three independent experiments. ${ }^{*} p<0.05$. ${ }^{* *} p<0.01$.

Paramyosin presents in thick myofilaments of invertebrate muscle and forms medullary rods surrounded by a cortical array of myosin rods [23]. In helminths, paramyosin serves not only as a structural protein but also as an immunomodulator. Earlier studies identified the expression of paramyosin in the tegument and on the surface of Taenia solium [24], Schistosoma mansoni, Schistosoma japonicum [25] and Fasciola hepatica [26]. It was observed that the paramyosin of helminth parasites could bind to human collagen [27-29], calgranulin [30], IgG [25,28,29], IgA [31], C1q [32], C8 [33] and C9 $[29,33]$, suggesting that surface-exposed paramyosin may play an important role as a potential modulator of the host immune system.

Our previous results showed that Ts-Pmy is present on the outer membrane of the cuticle of the adults and NBL of $T$. spiralis [16]. It was confirmed that surfaceexposed Ts-Pmy bound to complement C8 and C9, which are important components of the complement activation cascade and the membrane attack complex (MAC). The polymerization of $\mathrm{C} 9$, which was induced by $\mathrm{Zn}^{2+}$, was highly inhibited by $\mathrm{rTs}$-Pmy, indicating its interference in the assembly of the MAC during complement activation. The alternative complement pathway that activates the complement complex or polyC9 on rabbit erythrocytes $\left(\mathrm{E}_{\mathrm{R}}\right)$ was also inhibited by rTs-Pmy. Native $T s$-Pmy on the surface of $T$. spiralis effectively protected NBL from attack by the host complement system [14]. These results suggest that the outer membrane form of paramyosin expressed by $T$. spiralis had a role in host immunomodulation, presumably by inhibiting the formation of the MAC and thereby protecting the parasite from being attacked by the activated complement.

In this study, we further confirmed that native Ts-Pmy in the adult worm extracts was able to bind to human C9. In order to determine the C9 binding site on Ts-Pmy, different overlapped rTs-Pmy fragments were expressed as recombinant proteins and their ability to bind to C9 was tested. The results showed that the $\mathrm{C} 9$ binding region was located at the C-terminus of Ts-Pmy within position of 571-885, further narrowed down to the region between ${ }^{831}$ Leu and ${ }^{885}$ Tyr. To further map the complement C9 binding site within Ts-Pmy831-885, we prepared ten synthesized peptides covering the sequence of Ts-Pmy831885 with a few amino acids overlapping each other (P1P12). The result of the binding assay demonstrated that 15-amino acid peptides P8 (866-880) strongly bound to human C9. Chopping off one amino acid at C-terminus (P10) kept the same binding ability to C9, indicating that the C9 binding site on Ts-Pmy consists of 14 amino acid residues $\left({ }^{866} \mathrm{Val}-{ }^{879} \mathrm{Met}\right)$. Chopping off either ${ }^{866} \mathrm{Val}$ (P12) or ${ }^{879}$ Met (P11) lost the binding ability to $\mathrm{C} 9$, suggesting that both residues at both ends of the binding site, ${ }^{866} \mathrm{Val}$ and ${ }^{879}$ Met, are necessary for the binding affinity to human C9. A similar mapping study for the C9 binding site on paramyosin of Schistosoma mansoni (Sm-Pmy) only narrowed down to 123 amino acid residues in the $\mathrm{C}$-terminus [34]. Up to date, it is the first report to precisely locate the C9 binding site of paramyosin in helminths.

Functional analysis in this study revealed that peptide P10 ( ${ }^{866}$ Val- ${ }^{879}$ Met) was comparable to bind to C9 and further inhibit its function as full-length rTs-Pmy including C9 polymerization and complement-mediated $E_{R}$ lysis, suggesting that the binding site peptide could effectively inhibit the assembly of MAC and therefore protect the parasite from being attacked. Such results were consistent with the observations in the study on Sm-Pmy, which showed that C9 polymerization and hemolytic activity of human complement was inhibited by the C-terminal region of Sm-Pmy [34]. Therefore, the C-terminal regions of Ts-Pmy and Sm-Pmy have similar functions as complement inhibitors in capturing the complement component C9 to evade the first line of host's immune defense as a survival strategy. T. spiralis produces $T s$-Pmy through a 14 amino acids binding site to bind and neutralize $\mathrm{C} 9$ as a direct complement inhibitor.

$\mathrm{r} T s$-Pmy has been evaluated as a potential vaccine candidate antigen due to its complement neutralizing function $[13,14]$. The finding of the $\mathrm{C} 9$ binding domain on Ts-Pmy provides a good therapeutic target and a feasible approach to develop an epitope-based subunit vaccine against trichinellosis. A monoclonal antibody against the C9 binding domain has been produced and primary data 
with the antibody demonstrated protective immunity in passively transferred mice against $T$. spiralis larval infection (data not shown), indicating its potential as an epitope vaccine.

Our previous study revealed that another two epitopes on $T s$-Pmy recognized by protective monoclonal antibodies, one of which is located between ${ }^{88} \mathrm{Glu}$ and ${ }^{107} \mathrm{Glu}$ at the $\mathrm{N}$-terminus of $T s$-Pmy and another is a conformational epitope without a specific location, were also protective in immunized mice against $T$. spiralis larval challenge [19], indicating $T s$-Pmy may play multiple functions in the parasite life cycle, except for acting as an immunomodulator through neutralizing complement. A multi-epitope vaccine based on these two Ts-Pmy epitopes and another protective epitope from $T s 87$ produced higher levels of protection compared to the individual epitope vaccine [16]. However, the protection induced by these epitopes is not complete ( $35 \%)$. The addition of the C9 binding epitope identified in this study into the multiple epitope pipelines may increase the efficacy of protection against $T$. spiralis infection. The protective immunity of the Ts-Pmy C9 binding domain and its combination with other identified epitopes from $T$. spiralis vaccine pipelines against $T$. spiralis infection is under investigation.

\section{Conclusions}

This study mapped the C9 binding domain at the Cterminus between ${ }^{866} \mathrm{Val}$ and ${ }^{879} \mathrm{Met}$ of vaccine antigen $T s$-Pmy, which was capable of binding to C9 and preventing $\mathrm{C} 9$ polymerization. Our results revealed the precise molecular basis for T. spiralis utilizing Ts-Pmy as an immunomodulator to resist the attack of the host complement system. These results provide molecular evidence that $T$. spiralis evades host complement attacks by capturing and neutralizing complement components. This functional understanding of complement-evasion could serve as an important starting point for the development of site-targeting therapeutics and epitope-based subunit vaccines.

\section{Competing interests}

The authors declare that they have no competing interests.

\section{Authors' contributions}

$X Z$ performed the experiments and drafted the manuscript. YWH, JY, and YG performed some of the experiments. XPZ designed the study and revised the manuscript. All authors read and approved the final manuscript.

\footnotetext{
Acknowledgements

This study was supported by grants from the National Natural Science Foundation of China (81171598, 81371837), the National Science and Technology Major Project (2012ZX10004220-012) and the PhD Programs Foundation of the Municipal Education Commission of Beijing (20111002503). We thank Zhifei Zhang, Jingjing Huang, Xiaodi Yang, Xiaoqin Chen, Fengyun Wang and Jin Pan for their technical assistance.
}

Received: 6 January 2014 Accepted: 16 February 2014

Published: 24 February 2014

\section{References}

1. Dupouy-Camet J: Presidential address of ICT12 Conference: "Trichinella and trichinellosis-a never ending story". Vet Parasitol 2009, 159(3-4):194-196.

2. Wu Z, Sofronic-Milosavljevic L, Nagano I, Takahashi Y: Trichinella spiralis: nurse cell formation with emphasis on analogy to muscle cell repair. Parasit Vectors 2008, 1(1):27.

3. Pozio E: World distribution of Trichinella spp. infections in animals and humans. Vet Parasitol 2007, 149(1-2):3-21.

4. Pozio E, Gomez MM, Dupouy-Camet J: Clinical aspects, diagnosis and treatment of trichinellosis. Expert Rev Anti Infect Ther 2003, 1(3):471-482.

5. Pozio E, Rinaldi L, Marucci G, Musella V, Galati F, Cringoli G, Boireau P, La Rosa G: Hosts and habitats of Trichinella spiralis and Trichinella britovi in Europe. Int J Parasitol 2009, 39(1):71-79.

6. Dorny P, Praet N, Deckers N, Gabriel S: Emerging food-borne parasites. Vet Parasitol 2009, 163(3):196-206.

7. Gajadhar AA, Pozio E, Gamble HR, Nockler K, Maddox-Hyttel C, Forbes LB, Vallee I, Rossi P, Marinculic A, Boireau P: Trichinella diagnostics and control: mandatory and best practices for ensuring food safety. Vet Parasitol 2009, 159(3-4):197-205.

8. Zhang YL, Wang ZQ, Li LG, Cui J: Molecular characterization of Trichinella spiralis aminopeptidase and its potential as a novel vaccine candidate antigen against trichinellosis in BALB/c mice. Parasit Vectors 2013, 6(1):246.

9. Dunkelberger JR, Song WC: Complement and its role in innate and adaptive immune responses. Cell Res 2010, 20(1):34-50.

10. Lambris JD, Ricklin D, Geisbrecht BV: Complement evasion by human pathogens. Nat Rev Microbiol 2008, 6(2):132-142.

11. Kennedy MW, Kuo YM: The surfaces of the parasitic nematodes Trichinella spiralis and Toxocara canis differ in the binding of post-C3 components of human complement by the alternative pathway. Parasite Immunol 1988, 10(4):459-463.

12. Hong Y, Kim CW, Ghebrehiwet B: Trichinella spiralis: activation of complement by infective larvae, adults, and newborn larvae. Exp Parasitol 1992, 74(3):290-299.

13. Nareaho A, Saari S, Meri S, Sukura A: Complement membrane attack complex formation and infectivity of Trichinella spiralis and T. nativa in rats. Vet Parasitol 2009, 159(3-4):263-267.

14. Yang J, Yang Y, Gu Y, Li Q, Wei J, Wang S, Boireau P, Zhu X: Identification and characterization of a full-length CDNA encoding paramyosin of Trichinella spiralis. Biochem Biophys Res Commun 2008, 365(3):528-533.

15. Yang J, Gu Y, Yang Y, Wei J, Wang S, Cui S, Pan J, Li Q, Zhu X: Trichinella spiralis: immune response and protective immunity elicited by recombinant paramyosin formulated with different adjuvants. Exp Parasitol 2010, 124(4):403-408.

16. Zhang Z, Yang J, Wei J, Yang Y, Chen X, Zhao X, Gu Y, Cui S, Zhu X: Trichinella spiralis paramyosin binds to C8 and C9 and protects the tissue-dwelling nematode from being attacked by host complement. PLoS Negl Trop Dis 2011, 5(7):e1225.

17. Gamble HR, Bessonov AS, Cuperlovic K, Gajadhar AA, van Knapen F, Noeckler K, Schenone H, Zhu X: International Commission on Trichinellosis: recommendations on methods for the control of Trichinella in domestic and wild animals intended for human consumption. Vet Parasitol 2000, 93(3-4):393-408.

18. Gu Y, Wei J, Yang J, Huang J, Yang $X$, Zhu X: Protective Immunity against Trichinella spiralis Infection Induced by a Multi-Epitope Vaccine in a Murine Model. PLoS One 2013, 8(10):e77238.

19. Wei J, Gu Y, Yang J, Yang Y, Wang S, Cui S, Zhu X: Identification and characterization of protective epitope of Trichinella spiralis paramyosin. Vaccine 2011, 29(17):3162-3168.

20. Tschopp J: Circular polymerization of the membranolytic ninth component of complement. Dependence on metal ions. J Biol Chem 1984, 259(16):10569-10573.

21. Podack ER, Tschopp J: Circular polymerization of the ninth component of complement. Ring closure of the tubular complex confers resistance to detergent dissociation and to proteolytic degradation. J Biol Chem 1982, 257(24):15204-15212.

22. Yang $Y$, Zhang Z, Yang J, Chen X, Cui S, Zhu X: Oral vaccination with Ts87 DNA vaccine delivered by attenuated Salmonella typhimurium elicits a protective immune response against Trichinella spiralis larval challenge. Vaccine 2010, 28(15):2735-2742.

23. Gobert GN, McManus DP: Update on paramyosin in parasitic worms. Parasitol Int 2005, 54(2):101-107. 
24. Muhlschlegel F, Sygulla L, Frosch P, Massetti P, Frosch M: Paramyosin of Echinococcus granulosus: CDNA sequence and characterization of a tegumental antigen. Parasitol Res 1993, 79(8):660-666.

25. Loukas A, Jones MK, King LT, Brindley PJ, McManus DP: Receptor for Fc on the surfaces of schistosomes. Infect Immun 2001, 69(6):3646-3651.

26. Cancela M, Carmona C, Rossi S, Frangione B, Goni F, Berasain P: Purification, characterization, and immunolocalization of paramyosin from the adult stage of Fasciola hepatica. Parasitol Res 2004, 92(6):441-448.

27. Landa A, Laclette JP, Nicholson-Weller A, Shoemaker CB: CDNA cloning and recombinant expression of collagen-binding and complement inhibitor activity of Taenia solium paramyosin (AgB). Mol Biochem Parasitol 1993, 60(2):343-347.

28. Ferreira CA, Barbosa MC, Silveira TC, Valenzuela JG, Vaz ISJ, Masuda A: CDNA cloning, expression and characterization of a Boophilus microplus paramyosin. Parasitology 2002, 125(Pt 3):265-274.

29. Strube C, Buschbaum S, von Samson-Himmelstjerna G, Schnieder T: Stage-dependent transcriptional changes and characterization of paramyosin of the bovine lungworm Dictyocaulus viviparus. Parasitol Int 2009, 58(4):334-340.

30. Akpek EK, Liu SH, Thompson R, Gottsch JD: Identification of paramyosin as a binding protein for calgranulin $\mathrm{C}$ in experimental helminthic keratitis. Invest Ophthalmol Vis Sci 2002, 43(8):2677-2684.

31. Hernandez MG, Hafalla JC, Acosta LP, Aligui FF, Aligui GD, Ramirez BL, Dunne DW, Santiago ML: Paramyosin is a major target of the human IgA response against Schistosoma japonicum. Parasite Immunol 1999, 21(12):641-647.

32. Laclette JP, Shoemaker CB, Richter D, Arcos L, Pante N, Cohen C, Bing D, Nicholson-Weller A: Paramyosin inhibits complement C1.J I Immunol 1992, 148(1):124-128.

33. Deng J, Gold D, LoVerde PT, Fishelson Z: Inhibition of the complement membrane attack complex by Schistosoma mansoni paramyosin. Infect Immun 2003, 71(11):6402-6410.

34. Deng J, Gold D, LoVerde PT, Fishelson Z: Mapping of the complement C9 binding domain in paramyosin of the blood fluke Schistosoma mansoni. Int J Parasitol 2007, 37(1):67-75.

doi:10.1186/1756-3305-7-80

Cite this article as: Zhao et al:: Mapping of the complement $\mathrm{C} 9$ binding domain on Trichinella spiralis paramyosin. Parasites \& Vectors 2014 7:80.

\section{Submit your next manuscript to BioMed Central and take full advantage of:}

- Convenient online submission

- Thorough peer review

- No space constraints or color figure charges

- Immediate publication on acceptance

- Inclusion in PubMed, CAS, Scopus and Google Scholar

- Research which is freely available for redistribution 